\title{
COMPETENCIAS Y FORMACIÓN ÉTICA DE LOS PROFESIONALES DE LA COMUNICACIÓN CORPORATIVA EN CONTEXTOS INTERCULTURALES
}

\section{COMPETENCES AND ETHICAL TRAINING OF CORPORATE COMMUNICATION PROFESSIONALS IN INTERCULTURAL CONTEXTS}

(D) 8) Pablo Martín-Antoranz. Universidad Complutense de Madrid. España. pablomma@ucm.es

D 818 Francisco Cabezuelo-Lorenzo. Universidad Complutense de Madrid. España.

fcabezuelo@ucm.es

Ángel Bartolomé-Muñoz-de-Luna. Universidad San Pablo CEU. Madrid. España. abartolome@ceu.es

\section{RESUMEN}

Este trabajo propone una revisión de la necesidad de fomentar el estudio de la Ética y de la Deontología Profesional aplicada al ámbito de la Comunicación Estratégica e Institucional, sobre todo al mundo de las Relaciones Públicas y los Gabinetes de Comunicación. La revolución digital, que ha llegado para quedarse, plantea nuevos retos que el profesional de la comunicación estratégica debe afrontar: el uso de la información de los clientes, la privacidad y publicidad de las redes sociales, el nuevo derecho al olvido en la red, nuevos delitos a la intimidad y el honor a través de las redes sociales, la transparencia y acceso a la información tan de moda como herramientas para el control del poder político. A todo ello, hay que añadir el contexto de un nuevo escenario económico, social y cultural. Vivimos en una sociedad multicultural con conflictos étnicos, culturales y religiosos, permanentemente. En la actualidad, la interculturalidad se ha convertido en una realidad que no puede ser ignorada. Los futuros profesionales de la Comunicación necesitan una formación específica en valores universales que les capaciten para un mundo contemporáneo en el que cada vez tiene más sentido el término globalización. La ética adaptada a la interculturalidad es una competencia que debe desarrollarse de forma transversal en diferentes materias de las impartidas en los estudios, grados y especializaciones de las Ciencias de la Comunicación.

PALABRAS CLAVE: ética y deontología profesional; comunicación estratégica; gabinetes de comunicación; relaciones públicas; multiculturalidad.

\section{ABSTRACT}

This article proposes the necessary review of promoting the study of Ethics and Professional Deontology applied to the field of Strategic and Institutional 
Communication, especially to the world of Public Relations and Corporate Communication. The digital revolution, which has arrived to stay, poses new challenges that the professional of strategic communication must face, including the use of private information, the social media, the new right to be forgotten on the web, new crimes to privacy and honor through digital media, transparency and access to information. Also, we must add the context of a new economic, social and cultural scenario. We live in a multicultural society with ethnic, cultural and religious conflicts, permanently. At present, multiculturalism has become a reality that cannot be ignored. The upcoming professionals of Communication need a specific education based on universal values that enable them to find their place in a contemporary world in which the term globalization makes more and more sense. Ethics adapted to multiculturalism is a competence that must be developed transversally in different subjects from those taught in the studies, degrees and specializations of Communication Studies.

KEYWORDS: ethics and professional deontology; strategic communication; press office; public relations, multiculturalism.

\section{Cómo citar el artículo:}

Pablo Martín-Antoranz, Francisco Cabezuelo-Lorenzo y Ángel BartoloméMuñoz-de-Luna (2019). Competencias y formación ética de los profesionales de la comunicación corporativa en contextos interculturales. Revista de Ciencias de la Comunicación e Información, 24(1), 59-72.

doi: http://doi.org/10.35742/rcci.2019.24(1).59-72

\section{INTRODUCCIÓN Y JUSTIFICACIÓN}

Lo que es legal no siempre es ético y lo que es ético no siempre es legal, por tanto, la discusión sobre el papel que juega la ética dentro de la profesión de las Relaciones Públicas, es un tema que se ha discutido y continúa discutiéndose (Stortini, 2011). La actual crisis económica, política y social que viven las sociedades occidentales es el resultado, según muchos analistas, de una gran crisis de la ética y de la deontología profesional en diferentes áreas de actividad de nuestra sociedad digital, desde el mundo de la empresa, la economía al ámbito de las instituciones políticas y de los medios de comunicación. La Ética afecta a la comunicación corporativa, es decir, a la identidad, a la imagen y a la reputación. Nuestra "identidad" proviene de lo que nos entrega la sociedad en la que vivimos y que nos rodea. El filósofo asegura que somos producto de nuestro entorno. Por tanto, en la Ética influyen mucho nuestras tradiciones: aquellas costumbres y hábitos que nuestra sociedad ha ido adquiriendo con el paso del tiempo. A pesar del interés de esta cuestión, desde la óptica de la comunicación en las organizaciones, las investigaciones en este campo no son muy numerosas, aunque sí bastante interesantes, recientes y esperanzadoras. Casi todas ellas están basadas en estudios de Responsabilidad Social Corporativa (RSC).

Este trabajo intenta hacer hincapié no en las buenas prácticas de las organizaciones desde el punto de vista de la RSC, sino en el ejercicio de la labor profesional de los directores de comunicación de las diferentes organizaciones, ya sean éstas públicas 
o privadas, para lo cual se propone el estudio de los exitosos modelos de las principales asociaciones norteamericanas de profesionales de la comunicación. "La Ética queda establecida por lo que la empresa hace, no por lo que la empresa dice. Es necesario actuar de forma que sirva y que se vea que sirve al bienestar público", afirma Sam Black (1994, p. 196).

La inclusión de la ética de la comunicación en los tiempos de la interculturalidad implica una transformación en los planteamientos didácticos y exige un esfuerzo de adecuación pedagógica y de planificación de la que antes estaba alejada la docencia universitaria en la que esté presente la interculturalidad. Los futuros profesionales de la Comunicación necesitan una formación específica en valores universales que les capaciten para un mundo contemporáneo en el que cada vez tiene más sentido el término globalización. La ética adaptada a la interculturalidad es una competencia que debe desarrollarse de forma transversal en diferentes materias de las impartidas en los estudios, grados y especializaciones de las Ciencias de la Comunicación.

\section{OBJETIVOS Y METODOLOGÍA}

A la hora de establecer los objetivos, es preciso dejar claro desde un principio que, al igual que ocurre con muchos planteamientos filosóficos y éticos, este trabajo no pretende un estudio material ni cuantitativo de la realidad. Se podría decir que las palabras que siguen a continuación cuentan más con unas metas a largo plazo que con unos objetivos mensurables y cuantificables a corto plazo. El fin de este trabajo es hacer reflexionar a los investigadores en el campo de la comunicación corporativa sobre unos puntos y cuestiones que suelen pasar desapercibidos: los aspectos éticos y deontológicos del ejercicio profesional de la comunicación corporativa. Escándalos recientes como el de los Papeles de Panamá, las tarjetas tipo black-card de Bankia, diferentes escándalos políticos de todo tipo, la crisis de los refugiados sirios, o el caso de las emisiones contaminantes de Volkswagen, por ejemplo, ponen de manifiesto el valor de los fundamentos éticos. Los autores de este trabajo planteamos desde nuestra experiencia profesional, investigadora y docente algunas preguntas de investigación y proponemos como línea argumental en un primer momento hacer un repaso a la importancia de la Ética y su justificación en la poliédrica actividad comunicativa de las organizaciones. Seguidamente intentaremos dar respuesta y abrir nuevos interrogantes en relación a estas cuestiones de investigación. ¿Podemos considerar el oficio, trabajo u ocupación del comunicador en la Comunicación Corporativa como una profesión homogénea como tantas otras existentes en el mercado de trabajo y reguladas por la ley? ¿Sería necesario que los profesionales para ejercer su trabajo tuvieran que contar con una licencia o estar colegiados? ¿Es imprescindible un documento que nos acredite como profesionales de la comunicación? ¿Unos determinados estudios universitarios serían una garantía de la profesionalidad e integridad de los hombres y mujeres que trabajan en el sector?

Estos interrogantes son algunos de los aspectos que hoy en día se debaten nuestros colegas, especialmente en Estados Unidos y Canadá, pioneros en materia de Comunicación Empresarial e Institucional, y que, sin duda alguna, se han convertido en aspectos profesionales que también deben ser discutidos en España y resto de Europa. Ese es el fin que persigue y pretende cumplir este trabajo. Se busca un el 
debate sobre la correcta formación ética de los futuros profesionales de la Comunicación Corporativa. También se apuesta desde estas páginas por la implantación de códigos éticos efectivos y reales, no sólo simbólicos o ineficaces. Desde el punto de vista de la metodología, este trabajo propone una primera aproximación teórica al concepto y al estudio del ejercicio de la comunicación corporativa en la actual sociedad del conocimiento. Posteriormente, pasa a denunciar la falta de unos criterios éticos obligatorios y exige la omnipresencia de unos principios éticos y deontológicos en la profesión. Del mismo modo, propone unas claves propias para una Ética de la Comunicación Corporativa partiendo de la siguiente premisa: si la comunicación corporativa es un instrumento de gestión por medio del cual toda forma de comunicación interna y externa es conscientemente utilizada, con el fin de crear una base favorable para las relaciones con los públicos de los que la empresa depende, el conjunto de actuaciones del profesional de la comunicación debe responder a unas expectativas por parte de los stakeholders, que no pueden sentirse defraudados en la conducta de sus interlocutores. No hay imagen sin identidad, pues lo que se comunica no puede ser puro diseño, sino lo que verdaderamente trasciende y comunica es todo aquello anclado necesariamente en la realidad. Es cierto que una marca es una promesa, pero siempre debe ser una promesa basada en la realidad y con motivos más que justificados para atreverse a ligar el producto o servicio que promociona con la realidad. Cualquier otra intervención sería una mentira o tergiversación. Las actuales tendencias de estudios sobre reputación corporativa y también sobre responsabilidad social se fundamentan en el buen hacer y obrar de los miembros de una organización. Finalmente, fiel a la interés y servicio público, este trabajo propone el estudio de las organizaciones profesionales norteamericanas de la comunicación y sus códigos éticos, como son la PRSA o IABC, entre otros, para ofrecer un ejemplo de nuestros colegas americanos, que, en este aspecto, vuelven a liderar el mercado y son los auténticos creadores de las teorías de la responsabilidad social corporativa y el resurgir del debate sobre la necesidad de una formación de calidad para los futuros profesionales y el asociacionismo profesional, tan débil todavía en España, sobre todo después de la crisis económica que ha acabado con varias iniciativas en la materia puestas en marcha hace una década en los tiempos de supuesta bonanza económica.

\section{ANÁLISIS Y RESULTADOS}

En un primer momento, es necesaria una breve aproximación al concepto y ejercicio de la comunicación corporativa en la actual sociedad digital. La comunicación corporativa de una organización y el comportamiento ético de sus miembros son cosas diferentes, pero van de la mano, son interdependientes.

\subsection{UNA NECESARIA REVISIÓN DEL CLARO PERO AMPLIO CONCEPTO DE LA COMUNICACIÓN CORPORATIVA}

La comunicación corporativa, tal y como la entiende Cees B. M. Van Riel (1997, p. 26) es "un instrumento de gestión por medio del cual toda forma de comunicación interna y externa es conscientemente utilizada". Este instrumento se armoniza tan efectiva y eficazmente como sea posible con el fin de "crear una base favorable para las relaciones con los públicos de los que la empresa depende". Este concepto de comunicación corporativa, entendido como la gestión estratégica de todos los 
factores que influyen en la imagen de una organización, ha resumido durante los últimos años en nuestro país una visión estratégica de la imagen corporativa cuyo principal objetivo era armonizar las estrategias comunicativas y de negocio de las empresas (Villafañe 2004, 21). Esta primera aproximación conceptual a la comunicación corporativa ya señala, implícitamente, que una organización, sea del tipo que sea, no podrá tener una buena imagen ante sus públicos y stakeholders por muy buena que sea su identidad, si no la acompaña de buenas acciones, ya que no sólo el diseño comunica. Comunican también todas y cada una de las acciones de los miembros de una empresa o institución. Igual que Norberto Mínguez (1999) afirma que "los conceptos de identidad e imagen corporativa son interdependientes" y que "no hay imagen sin identidad, pues lo que se comunica no puede ser puro diseño, sino que ha de estar anclado necesariamente en la realidad". Tampoco es difícil coincidir con Mínguez cuando señala que

la imagen corporativa no atañe exclusivamente al departamento de comunicación, sino que es una tarea que afecta a la alta dirección y su gestión exige el compromiso de las distintas áreas funcionales de la organización y, en última instancia, de todos y cada uno de los empleados de la empresa.

Efectivamente, una imagen y una reputación positivas no son sólo el resultado de una buena comunicación, sino de una actividad conforme a lo que esperan los públicos de una organización.

Aunque la comunicación corporativa es hoy en día algo vital, no es el único elemento que aportará una buena reputación a la organización. Empresas e instituciones deben hoy gestionar de forma eficaz y sistemática todos sus activos, con especial hincapié en aquellos que una vez que se pierden no se puede recuperar con facilidad. Como afirma Mínguez, "la imagen debe gestionarse en todos los niveles de la organización y en todas las áreas de su actividad, pues el comportamiento de la empresa, cada acto de cada uno de sus empleados, tiene un efecto en su imagen global". Llegamos a otra nueva realidad actual: la etapa de los estudios de reputación corporativa. Se entiende por "reputación corporativa" la expresión de la identidad de la organización y del reconocimiento de su comportamiento corporativo. Para Justo Villafañe (2004, p. 29), la identidad corporativa es la síntesis de la historia de la organización, de su estrategia o proyecto empresarial vigente y de su cultura corporativa. Desde esta perspectiva, para este autor, la noción de identidad es sinónimo de realidad corporativa y la reputación tiene "su origen en la realidad de la empresa y, más concretamente, en su historia, en la credibilidad del proyecto empresarial vigente y en la alineación de su cultura corporativa con ese proyecto". Así, Villafañe define la reputación como "la cristalización de la imagen corporativa de una entidad cuando ésta es el resultado de un comportamiento corporativo excelente, mantenido a lo largo del tiempo, que le confiere un carácter estructural ante sus stakeholders estratégicos" (Villafañe 2004, p. 2). Esta comunicación corporativa que persigue la buena reputación no es un concepto abstracto.

\subsection{PLANTEAMIENTOS ÉTICOS EN LA ACTUAL SOCIEDAD DIGITAL}

Sin duda alguna, resulta bastante difícil poder determinar con claridad hacia dónde nos lleva la actual realidad postmoderna. En este primer cuarto del siglo XXI, con 
Donald Trump como presidente de Estados Unidos, los discursos intelectuales y políticos han perdido credibilidad, nuestro mundo postmoderno sufre un proceso de "atomización" y de nuevos movimientos populistas. Así, parece que los actuales planteamientos éticos en nuestra sociedad de la información y el conocimiento se sitúan a caballo entre el escepticismo de algunos autores griegos de la Antigüedad y el saludable pragmatismo aristotélico, por lo que algunos autores se atreven a afirmar que hoy la mayor parte de los ciudadanos occidentales dudemos de quienes declaran que la verdad existe y afirman tener vinculación directa con ella. Las pistas parecen ir por otros derroteros. En el siglo XX, tras grandes fracasos históricos, heredamos, sin embargo, algunos valores positivos, como aquellos lanzados por los movimientos de preocupación por la cuestión de nuestra relación con el entorno natural. La tendencia actual acostumbra a ligar los estudios de comportamiento del ser humano con su entorno.

Ya en la Antigüedad, Aristóteles denominaba al ser humano como un "animal político" (zoon politikon). Los seres humanos somos seres sociales por naturaleza. Necesitamos de los demás. Necesitamos de la comunicación. Para Aristóteles, nuestra "identidad" proviene de lo que nos entrega la sociedad en la que vivimos y que nos rodea. Aristóteles asegura que somos producto de nuestro entorno. Los seres humanos somos extraordinariamente gregarios. La vida comunitaria se sustenta en tradiciones y en valores que los grupos entregan a sus miembros. Aristóteles proponía que se debe incentivar actitudes positivas hacia otros, luego, el comportamiento ético se volverá instintivo, independiente de reglas o sistemas. Por tanto, en la Ética influyen mucho nuestras tradiciones, aquellas costumbres y hábitos que nuestra sociedad ha ido adquiriendo con el paso del tiempo.

Filósofos anteriores como Platón ya habían descrito la preocupación existente por ese entonces sobre algunos asuntos morales en el Mundo Antiguo, sentando, de este modo, las bases de lo que actualmente denominamos el "análisis ético". Platón ya era consciente de que todos tenemos nuestras propias ideas de lo que es "bueno" y lo que es "malo". Los tiempos cambian y muchos autores se lanzar a afirmar hoy que la Ética de la Comunicación se encuentra en una encrucijada.

Por tanto, la pregunta es la siguiente. ¿Deben cambiar nuestros principios éticos en la actual sociedad de la información y el conocimiento? ¿La Ética se adapta a los tiempos? Sin duda alguna, los valores y criterios directores de nuestras actuaciones tienen que adaptarse a los tiempos. Nuestros valores y criterios se ven afectados en cierta forma por la evolución de los propios conocimientos que tan rápidamente se propagan gracias a las nuevas tecnologías de la actual sociedad de la información. Sin embargo, esta evolución no significa que todo esté cambiando constantemente en el campo de la Ética ni que estos valores puedan ser "relativizados".

Algunos autores, como Enebral Casares (1995), nos recuerdan que los criterios encuadrados en la Ética pretenden "el mejor, más auténtico y más sostenido modo de regular las interacciones que están teniendo efecto en un grupo por la vitalidad de sus componentes". Se entiende, por tanto, que hay un ánimo axiomático que seguirá siendo siempre válido en toda circunstancia, es decir, la Ética ha estado, está y estará ahí para intentar mejorar, hacer más auténtico, sostenido y eficaz el modo de regular las interacciones que tienen lugar entre los diferentes miembros de un grupo. 
Ése es el fundamento permanente y siempre válido de la Ética. Así, se puede afirmar que las manifestaciones concretas de ese principio inspirador y fundamental de la Ética pueden y deben ir adaptándose a las propias interacciones a las que se aplique. Enebral Casares (1995) nos recuerda también que

cuando la Humanidad se restringía a grupúsculos tribales, la Ética en ellos tenía expresiones apropiadas a su nivel de conocimientos y de la complejidad social de su civilización. Pero, cuando éstos varían, aumentan, y se enriquecen con nuevas y múltiples facetas, la Ética deberá proponerse abarcar estos nuevos aspectos de una manera coherente con el caudal de conocimientos que ahora se tengan sobre la propia complejidad que manifiesta la convivencia.

Hoy vivimos en la sociedad de la información y del conocimiento, de las nuevas tecnologías, por lo que la Ética exige una aplicación determinada.

La Ética en sí misma está siempre en continua evolución en tanto en cuanto se está desarrollando y poniendo en práctica constantemente, pero eso no significa que sea un "valor relativizado". Dicho de otro modo, la Ética está en evolución, pero no los principios que la inspiran, es decir, mantiene su eje axiomático consistente en mejorar, autentificar y garantizar el sostenimiento de las interacciones justa y eficazmente. Esos principios han sido, son y serán siempre válidos, e inspiradores a su vez de cada uno de los desarrollos que adquiera su adecuación a los nuevos eventos en los que se aplique (Cabezuelo Lorenzo \& Pérez Serrano, 2008, p. 10).

De este modo, no podemos decir que debido a los cambios en esta sociedad, cada vez más compleja, y especialmente en las interacciones que en ella se producen, la Ética ya no existe o ha desaparecido. Nunca podrá decirse que la Ética está en crisis, al menos si entendemos como tal la exigencia connatural de la inteligencia humana para procurar gobernar mejor el entorno en el que vive. A día de hoy, han cambiado las aplicaciones que se hacen de la Ética. Es decir, han cambiado el conjunto de criterios referenciales de segundo orden, más pragmáticos, y más circunscritos a determinadas perspectivas. Estos criterios sí pueden estar en crisis y pueden necesitar una renovación o actualización drástica. Hoy en día, el lado más pragmático e inmediato de la Ética no podría ser probablemente entendida en otro contexto temporal. Esta necesidad de contar con unos principios éticos, además de con unos valores en la profesión, resulta particularmente interesante en uno de los campos de especialización de la comunicación aplicada, como es el caso del ejercicio profesional de la Comunicación Corporativa.

\subsection{UNA ÉTICA PROPIA DE LA COMUNICACIÓN CORPORATIVA}

Joan Costa habla del "lenguaje de los actos". Para el autor catalán este lenguaje corporativo debe guardar una coherencia necesaria y obligatoria con el resto de mensajes que la organización intercambia con sus stakeholders. Este lenguaje de los actos se basa en la convicción, el compromiso y la autorregulación de todos los integrantes de la organización. En el caso de las grandes organizaciones, como pueda ser el caso de las gigantes y omnipresentes empresas multinacionales, esos grupos de interés están conformados principalmente por los accionistas, los inversores, los mercados financieros en los que cotizan sus acciones y los 
organismos reguladores. También podemos incluir el propio personal y sus familias, contemplados no sólo en su relación directa con la empresa sino también en sus respectivos ámbitos sociales. Por supuesto, no pueden faltar los clientes, consumidores o usuarios ni los proveedores. En esas empresas, caracterizadas por su compleja organización y diversidad de públicos, la comunicación con cada uno de esos segmentos constituye en sí misma una especialidad necesariamente a cargo de distintas áreas y departamentos, pero siempre con una toma de decisiones que tenga como paraguas común la Ética y Deontología profesional de la Comunicación (Cabezuelo Lorenzo \& Pérez Serrano, 2008, p. 12).

Sam Black (1993, p. 195) afirma que "la declaración de objetivos de una empresa debería proporcionar una orientación sobre qué tipo de comportamiento ético es obligatorio dentro de la organización" y que "el concepto de valores éticos y morales en una empresa debe proceder de lo alto e irse reflejando hasta llegar a lo más bajo". Sin duda alguna, la cuestión de la Ética en la comunicación es una preocupación de vital importancia para aquellos profesionales de la comunicación corporativa que trabajan día a día por lograr la mejor aceptación y apoyo del público para sus organizaciones.

Los valores morales y éticos no son siempre absolutos. Además, su articulación en cualquier empresa o institución estará relacionada, en todo momento, con la cultura corporativa de la organización, pero no lo puede estar por las campañas o estrategias varias y sus tácticas, que buscan conseguir objetivos a corto plazo.

Melvin Sharpe, profesor de la Ball State University of Indiana, formula las siguientes pautas para comunicar de forma ética y profesional:

a) Comunicar honestamente con el fin de obtener credibilidad.

b) Ser abierta y consistente en todas sus acciones con el fin de obtener la confianza del público.

c) Mantener la imparcialidad y equidad en sus actos con el fin de ofrecer un trato justo.

d) Mantener una comunicación continua con el fin de que lograr respeto y comprensión mutuos.

e) Investigar de forma adecuada su entorno social con el fin de comunicar sus posturas de una forma más eficaz y que cambie sus acciones, cuando éstas ya no sirvan al interés público.

Sharpe también considera que las decisiones tomadas a escondidas, aunque procedan de la Ética, llevan la mancha de la desconfianza. La realidad y la imagen deben entrelazarse; no sólo debe hacerse justicia, debe verse que se hace. Este aspecto es de una importancia vital para la comunicación corporativa.

\subsection{EL ASOCIACIONISMO DE ORIGEN ANGLOSAJÓN COMO PROPUESTA PARA LA AUTORREGULACIÓN}

Desde el punto de vista deontológico, se considera que los investigadores en ciencias de la comunicación, al igual que en cualquier otra ciencia, deben actuar en función de un código deontológico (Parés i Maicas, 2006, p. 46). 
En algunos países de nuestro entorno, especialmente en aquellos más avanzados en materia de comunicación, existen, desde hace muchos años, organizaciones profesionales que pueden ser muy útiles para orientar en su trabajo diario a los profesionales de la comunicación. En España, cada vez más, surgen varias iniciativas de este tipo, con el fin de defender los derechos de estos colectivos. Este artículo propone una revisión de las posturas de las principales asociaciones profesionales internacionales en las que se agrupan miles de profesionales de la comunicación para extraer unas conclusiones y pautas claras que sirvan para crear una Ética propia de la Comunicación Corporativa. The Public Relations Society of America (PRSA), The Institute of Public Relations (IPR), The International Association of Business Communication (IABC) son algunas de las asociaciones aquí analizadas.

The Public Relations Society of America (PRSA) es la asociación de Relaciones Públicas más grande de todo el mundo. Tiene su sede en Nueva York y un total de 116 delegaciones en todo el territorio estadounidense. A su vez, se divide en 18 secciones profesionales que van desde el área de negocios e industria, hasta firmas como consultoras, profesionales independientes, organizaciones sin ánimo de lucro, instituciones académicas y un largo etcétera. Celebra una importante reunión o conferencia anual y saca a la luz dos publicaciones, una titulada 'Tactics', un tabloide mensual con información sobre la profesión, y 'The Strategist', una revista que analiza más en profundidad los cambios en la práctica de las Relaciones Públicas. La PRSA basa sus valores profesionales en el buen consejo, la honestidad, la experiencia, independencia, lealtad y rectitud de sus miembros, e invita a sus miembros a servir al interés público, ofreciendo una sola voz para un debate de ideas bien informado. Busca la adhesión de sus miembros a los más altos estándares de exactitud y veracidad para servir, así, provechosamente a los intereses de sus clientes y a la comunicación con sus públicos y audiencias. El código ético propuesto por la PRSA se basa en seis valores que considera "vitales para la integridad de la profesión en su conjunto" y afirma que "el valor de la reputación de los socios depende de la conducta ética de cada afiliado de la PRSA". "Cada uno de nosotros es un ejemplo para los demás, así como para otros profesionales, al procurar excelencia en nuestra labor con intensas normas de desempeño, profesionalismo y conducta ética", añade su código. La PRSA invita a servir al interés público, ofreciendo un debate de ideas bien informado adhiriéndose a los más altos estándares de exactitud y veracidad para los intereses de sus clientes y la comunicación con el público, al tiempo que generan un entendimiento mutuo en la actual sociedad del conocimiento.

En el caso de la PRSA, a pesar de que en teoría es uno de los mejores cuadros para orientar la profesión, encontramos que, a pesar de los numerosos escándalos, su estricto cumplimiento ha quedado bastante limitado. Así, por ejemplo, encontramos que desde 1990 a 1998 ningún miembro de la PRSA fue recriminado por acción alguna, ni menos expulsado o suspendido de la asociación.

La segunda gran asociación de los profesionales de la comunicación es The International Association of Business Communicators (IABC). Tiene su sede en San Francisco (EEUU). Cuenta con miembros distribuidos a lo largo de más de 60 países. Aunque la mayoría de ellos están en Estados Unidos, cuenta con secciones 
muy importantes en Canadá, Reino Unido y Hong Kong. Entre las actividades de la IABC destaca la publicación de la prestigiosa revista 'Communication World', en la que analiza en profundidad algunos casos prácticos e informa de las últimas novedades en el sector. Su código se basa en tres principios que vienen a decir que la comunicación profesional debe ser, ante todo, legal, ética, y de buen gusto.

The Internacional Public Relations Association (IPRA) es la tercera organización profesional más importante en el campo de las Relaciones Públicas. Es también anglosajona, pero escapa ya de Norteamérica. La IPRA tiene su sede en Londres (Reino Unido) y cuenta con miembros repartidos en un total de 96 países por todo el mundo. La mayoría de sus miembros podrían ser definidos como "senior international public relations executives". Su publicación principal lleva por título 'Frontline'.

PRSA, IABC e IPRA son las organizaciones que abarcan a un mayor número de profesionales (Wilcox \& Cameron, 2006), pero hay otras tantas entidades bastante importantes y que han ejercido un papel destacado en la implantación de códigos éticos entre los norteamericanos. Sin duda alguna, una de las cosas que primero llama la atención al estudiar la Ética aplicada a la Comunicación en Norteamérica es el uso de los nombres, es decir, la terminología. La propia forma de referirse a los códigos como (codes of conduct) en vez de "código ético o deontológico" ya nos da una muestra más del pragmatismo norteamericano. Prácticamente, cada asociación tiene su propio código, siendo especialmente conocidos los de las organizaciones anteriormente citadas, y la mayoría de ellas se reserva el derecho de criticar, censurar, o expulsar de su seno a quien no cumpla con estos códigos. El código de la IABC hace un especial hincapié en subrayar el principio de que el profesional de la comunicación debe ir más allá de lo legal y lo ético, debe también apostar por el buen gusto y los valores y creencias culturales más decorosas. IABC basa sus principios en la legalidad, la ética y el buen gusto, en un código de doce artículos en los que se subraya la necesidad de que los profesionales practiquen una comunicación honesta, verdadera y plural, al promover la libre circulación y flujo de información y contenidos de acuerdo con el interés general del público. Esta organización también pone énfasis en el compromiso de los profesionales con la verdad y la libertad de expresión, además del respeto por la legalidad vigente. Igualmente presta especial atención al respeto a los valores y creencias culturales, el pluralismo. También alienta la corrección de errores detectados, a la vez que exige confidencialidad de la información a la que tienen acceso por su actividad.

\subsection{LA INTERCULTURALIDAD, UNA PROPUESTA PARA LA MEJORA DE LA COMUNICACIÓN GLOBAL}

En general, la interculturalidad se presenta como un nuevo reto en las aulas universitarias de Ciencias de la Comunicación y de la Información. En las aulas universitarias de Estados Unidos, en la materia de Ética de la Comunicación, uno de los temas claves es el de la interculturalidad y respeto a la diversidad. La interculturalidad debe ser entendida como un principio, pero también como un proyecto para la mejora de las acciones y relaciones entre las culturas. En el plano didáctico, se configura mediante el diálogo y el encuentro compartido entre todos los estudiantes, el profesorado y las comunidades participantes. La interculturalidad 
debe buscar el encuentro creativo de experiencias humanas diversas. Además, necesita nuevas concepciones y prácticas educativas centradas en la equidad, la solidaridad y el acercamiento plural entre todas las personas y pueblos.

En el nuevo Espacio Europeo de Educación Superior (EEES), conocido popularmente como Bolonia, se sitúa como una de las competencias transversales más importantes. La interculturalidad se ha convertido en una realidad que no puede ser ignorada. Esta realidad ha llegado a las aulas de centros de primaria y secundaria pero también a las de las facultades y centros de postgrado vinculados a las Ciencias de la Información y la Comunicación. Pero, ¿qué es la interculturalidad? ¿Cuenta el docente con las herramientas necesarias para interiorizarla en su ritmo didáctico?

La interculturalidad es "el principio y proyecto para la mejora de las acciones y relaciones entre las culturas, configurada mediante el diálogo y el encuentro compartido entre todos los estudiantes, el profesorado y las comunidades participantes" (Domínguez, 2006, p. 12); o, dicho de otro modo,

la síntesis superadora y vivencial del ser y saber humano que se reconoce a sí mismo en la relación y el encuentro creativo de experiencias humanas diversas, que necesita nuevas concepciones y prácticas educativas centradas en la equidad, la solidaridad y el acercamiento plural entre todas las personas y pueblos, con el máximo respeto a la identidad en el horizonte de nuevas actuaciones de carácter global" (Medina et al., 2004).

Este planteamiento de apertura y entendimiento se incardina perfectamente en la interdependencia que exige el mundo en el que vivimos que, sea en el contexto que sea, motiva la colaboración entre ciudadanos de países antes aislados. Como profesores universitarios, hemos vivido esta realidad gracias a la implantación de forma experimental y avanzada de nuestros estudios conforme al EEES y a diferentes experiencias de intercambio académico (de alumnos, profesores y personal no docente) gracias al ya asentado programa Erasmus. Esta concepción intercultural ha llegado a la universidad $y$, aunque no sin dificultades, se está traduciendo en estrategias que persiguen la consecución de objetivos como la formación de ciudadanos libres a través de un sistema educativo de calidad, principio que se sitúa como uno de los valores positivos con los que se identifica nuestra civilización.

La incursión de la interculturalidad en la realidad y práctica docentes es un campo muy tratado y motiva profusamente la preocupación constante de profesores, pedagogos e investigadores. Lo que no es tan habitual es que se vincule a la docencia universitaria y, menos aún, a la especificidad de los estudios de Comunicación.

Aunque durante muchos años, España poseía una realidad diferente respecto a otros países de su entorno europeo, pero poco a poco nuestra realidad sociodemográfica ha dejado de ser peculiar (Cabezuelo Lorenzo \& Pérez Serrano, 2009, p. 9). La inmigración empezó a crecer a en los años noventa con fuerza y se ha incrementado de manera exponencial en la última década y media, hasta llegar a situarse, dentro del espectro docente, como un reto creciente. Así, ahora, es más 
que evidente la presencia de alumnos de diferentes nacionalidades en las aulas españolas, que motiva -en algunas ocasiones- la aparición de un abanico de situaciones complejas planteadas por los discentes que, en algunas ocasiones, tienen que ver con una plausible carencia idiomática e, incluso, una deficiente escolarización previa.

Diferentes estudios sobre interculturalidad y multiculturalidad marcan que, la primera se refiere a la interacción y comunicación entre diferentes culturas, de una forma respetuosa, horizontal y sinérgica, donde no se concibe que un grupo cultural pueda estar sobredimensionado con respecto a otro y donde se favorece, en todo momento, la integración y convivencia de ambas partes. En las relaciones interculturales se establece un vínculo basado en el respeto a la diversidad y el enriquecimiento mutuo, algo que es vital en estos tiempos, y más si cabe en estos de crisis y conflictos. La formación en cuestiones interculturales enseña que los conflictos se resuelven a través del respeto, el diálogo, la escucha mutua, la concertación y la unión de buenas voluntades (Cabezuelo Lorenzo \& Pérez Serrano, 2009, p. 10).

En la actualidad, la interculturalidad se ha convertido en una realidad que no puede ser ignorada. Esta realidad ha llegado a las aulas, por eso desde el campo académico se necesitan nuevas concepciones y prácticas educativas centradas en valores universales, con el máximo respeto a la pluralidad del actual mundo globalizado en el que vivimos. La inclusión de la interculturalidad en la realidad y práctica docentes es un campo muy tratado y motiva profusamente la preocupación constante de profesores, pedagogos e investigadores. Lo que no es tan habitual es que se vincule a la docencia universitaria y, menos aún, a la especificidad de los estudios de las Facultades de Ciencias de la Comunicación.

Los futuros profesionales de la Comunicación que actualmente se forman como alumnos en las titulaciones de Periodismo, Publicidad y Relaciones Públicas necesitan una formación específica en valores universales que les capaciten para un mundo contemporáneo en el que cada vez tiene más sentido el término globalización. La interculturalidad es una competencia que debe desarrollarse de forma transversal en diferentes materias de las impartidas en los estudios, grados y especializaciones de las Ciencias de la Comunicación.

\section{CONCLUSIONES}

Al fin y al cabo, todas las organizaciones son entes neutros, aparentemente, pero están formados por un conjunto de personas, por tanto, no son morales ni inmorales, más bien podemos decir que son amorales, pero sí puede ser moral o inmoral el comportamiento de sus miembros que, en ese momento, son identificados con la corporación a la que representan. Por eso, los profesionales de la comunicación corporativa deben entender el valor real y significado del ejercicio profesional conforme a unas pautas, lo que sin duda alguna redundaría en beneficio de la Ética. Por eso, este trabajo defiende la necesidad de la autorregulación profesional a través a asociaciones e institutos profesionales, promoviendo el asociacionismo profesional ordenado a imitación del modelo americano basado en tres grandes ejemplos asociativos profesionales. 
Los últimos casos de corrupción, falsedad o fraude, desde el caso de las emisiones de Volkswagen al de los Papeles de Panamá, ponen de manifiesto, además de la necesidad de mantener las exigencias éticas, especialmente en el campo de la Comunicación Corporativa, Institucional y Relaciones Públicas, la necesidad de cuestionarnos la importancia de los valores éticos en el trabajo diario. La Comunicación Corporativa, tanto en Norteamérica como en Europa, debe encontrar en la Ética el eje del ejercicio de la actividad profesional de forma que los códigos no suplanten a las instancias jurídicas, ni supongan una autocensura, pero que sí cuenten con la fuerza suficiente como poder fiscalizador efectivo que colabore notablemente con mejorar la buena reputación.

Las organizaciones, ya sean empresas o instituciones, deben ir más allá de los derechos y obligaciones que les corresponden como entes jurídicos. Además de cumplir con los fines específicos para los que fueron creados, también pueden ir más allá y desarrollar su actividad como ciudadanos corporativos comprometidos con la comunidad que les rodea o en la que desarrollan su actividad. Esta relación define los alcances de su responsabilidad social a partir del presupuesto único de una conducta ética basada en una clara enunciación de principios y valores compartidos por toda la organización, y en el ejercicio de la autorregulación como guía de su forma de proceder en todos los aspectos. La ética y la responsabilidad social son temas vitales en las Relaciones Públicas, dado que en su esfuerzo deliberado, planificado y continuo por establecer y mantener una comprensión mutua, facilitan la comunicación entre la empresa y sus diferentes audiencias clave, incluyendo a la sociedad en general (Stortini, 2011).

Es preciso cuestionarnos más a menudo sobre los aspectos éticos, deontológicos y profesionales de la comunicación. A menudo, pensamos sólo en la eficacia y la rentabilidad, fruto de nuestra mentalidad productiva. En Norteamérica, esa parte del mundo tan criticada por su mentalidad de consumo, se habla más de ética que en Europa y se usan permanentemente estos términos cuando se habla de buenas prácticas en materia de Comunicación Corporativa. Las compañías americanas dedican más a causas sociales que las europeas y los profesionales americanos han estado otra vez más atentos a temas de atención a la diversidad, sobre todo en cuestiones de interculturalidad y multiculturalidad. Hoy en día en Norteamérica no se concibe que un grupo cultural pueda estar sobredimensionado con respecto a otro y donde se favorece, en todo momento, la integración y convivencia. En toda relación intercultural hay un vínculo basado en el respeto a la diversidad y el enriquecimiento mutuo. La formación en cuestiones interculturales enseña que los conflictos se resuelven a través del respeto, el diálogo, la escucha mutua, la concertación y la unión de buenas voluntades. Estas competencias deben ser introducidas en el currículum de los grados de Ciencias de la Comunicación a través de las materias de Ética de la Comunicación y similares. En la actualidad, la interculturalidad se ha convertido en una realidad que no puede ser ignorada y debe ser objeto de estudio en las aulas, por eso desde el campo académico se necesitan nuevas concepciones y prácticas educativas centradas en valores universales, con el máximo respeto a la pluralidad del actual mundo globalizado en el que vivimos. La inclusión de la interculturalidad es vital.

Estos son algunos de los aspectos que hoy en día se debaten nuestros colegas norteamericanos y anglófonos, sociedades pioneras en la puesta en marcha de ese 
famoso Mix, y que, poco a poco en España se estudian del mismo modo, debido al mundo globalizado que compartimos. Sin duda alguna, todas estas cuestiones se han convertido en aspectos profesionales que también deben ser discutidos en España y en el resto de Europa. Un primer paso en esta línea, es el fin que persigue este trabajo: el debate sobre la correcta formación ética de los futuros profesionales de la comunicación corporativa a través de valores éticos como son la Multiculturalidad y la Interculturalidad.

\section{REFERENCIAS BIBLIOGRÁFICAS}

Black, S. (1994). ABC de las Relaciones Públicas. Barcelona: Gestión 2000.

Cabezuelo Lorenzo, F. y Pérez Serrano, M. J. (2008). Aspectos éticos y deontológicos del ejercicio profesional de la comunicación corporativa. Icono 14, 6(2).

Cabezuelo Lorenzo, F. y Pérez Serrano, M. J. (2009). Estudio de la interculturalidad como competencia transversal en las aulas de Ciencias de la Comunicación. Vivat Academia, Revista de Comunicación, (108), 1-25. doi: http://dx.doi.org/10.15178/va.2009.108.1-25

Domínguez Garrido, M. C. (2006). Investigación y formación del profesorado en una sociedad intercultural. Madrid: Ed. Universitas.

Enebral Casares, F. (1992). La información interactiva en gabinetes de comunicación (tesis doctoral). Facultad de Ciencias de la información. Universidad Complutense de Madrid.

Medina Revilla, A., Rodríguez Marcos, A. e Ibáñez de Aldecoa, A. [Coords.] (2005). Interculturalidad: Formación del Profesorado y Educación. Madrid: Ed. Pearson Prentice Hall.

Mínguez, N. (1999). Un marco conceptual para la comunicación corporativa. Revista ZER. Universidad del País Vasco, 7.

Parés i Maicas, M. (2006). Las relaciones públicas, una ciencia social. Anàlisi: Quaderns de Comunicació i Cultura, (34), 23-48.

Sharpe, M. (1990). International Public Relations Review, 13(3), 21-25.

Stortini, G. P. (2011). La ética en las Relaciones Públicas. Cuadernos del Centro de Estudios en Diseño y Comunicación. Ensayos (Argentina), (35), 117-127.

Van Riel, C. B. M (1997). Comunicación Corporativa. Madrid: Prentice Hall.

Villafañe, J. (2004). La buena reputación. Claves del valor intangible de las empresas. Madrid: Editorial Pirámide.

Wilcox, D. \& Cameron, G. (2006). Public Relations: Strategies and Tactics. 8th edition. Boston: Allyn and Bacon. 